\title{
Belphégor
}

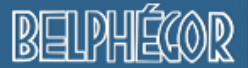

Littérature populaire et culture médiatique

18-2 | 2020

Roman Populaire Espagnol - Roman historique, 1900-1950

\section{Littérature de genre et écriture spéculaire chez Gonzalo Suárez}

\section{Boris Monneau}

\section{OpenEdition}

Journals

Édition électronique

URL : http://journals.openedition.org/belphegor/3198

DOI : $10.4000 /$ belphegor.3198

ISSN : 1499-7185

Éditeur

LPCM

Référence électronique

Boris Monneau, «Littérature de genre et écriture spéculaire chez Gonzalo Suárez », Belphégor [En ligne], 18-2 | 2020, mis en ligne le 11 décembre 2020, consulté le 28 avril 2021. URL : http:// journals.openedition.org/belphegor/3198; DOI : https://doi.org/10.4000/belphegor.3198

Ce document a été généré automatiquement le 28 avril 2021.

\section{(c)}

Belphégor est mis à disposition selon les termes de la Licence Creative Commons Attribution - Pas d'Utilisation Commerciale - Pas de Modification 4.0 International. 


\title{
Littérature de genre et écriture spéculaire chez Gonzalo Suárez
}

\author{
Boris Monneau
}

1 L'œuvre littéraire de Gonzalo Suárez n'appartient pas réellement au champ de la littérature populaire. Tout comme son cinéma, apparenté au versant le plus avantgardiste de la nouvelle vague espagnole que fut l'Ecole de Barcelone ${ }^{1}$, ses livres ont pu être considérés par Javier Cercas comme «des jalons qui signlaient le chemin d'une modernité à laquelle la littérature espagnole luttait pour s'incorporer ${ }^{2}$ » et Robert Saladrigas définit son premier roman, De cuerpo presente (1963) comme «la première tentative sérieuse et absolument valable de roman avant-gardiste espagnol de l'aprèsguerre $^{3}$ ». L'élite intellectuelle et universitaire barcelonaise fut selon Cercas le premier public de Suárez ${ }^{4}$. Son œuvre a effectivement été saluée par Pere Gimferrer, mais encore par Vicente Aleixandre, Max Aub ou Julio Cortázar.

Cependant, ses romans et récits se composent de nombreux emprunts à la littérature de genre. Lui-même déclare dans la préface à l'anthologie Las fuentes del Nilo : «Opinaba que las obras maestras y sus sucedáneos nos amueblan la casa, pero los llamados géneros menores nos abrían las ventanas ${ }^{5}$ ", citant la prégnance imaginaire d'écrits tels que Frankenstein, Jekyll and Hyde ou Tarzan, leur capacité à faire exister des figures en dehors même des livres. Puisque ses œuvres se présentent pour la plupart comme des variations autour de genres liés à la littérature populaire, et qu'elles font un usage abondant de références à la culture de masse et aux moyens de communication ${ }^{6}$, dans une démarche relevant parfois du collage, Javier Cercas a pu définir à juste titre Gonzalo Suárez comme un "écrivain pop $^{7}$ ", lui-même utilisant ce qualificatif pour définir De cuerpo presente ${ }^{8}$. Mais il ne s'agît pas seulement de refléter le monde en adoptant ses codes d'écriture : c'est l'écriture elle-même qui est pensée à partir du genre. Nous étudierons ainsi l'usage du genre littéraire dans les œuvres de Gonzalo Suárez comme le catalyseur d'une écriture spéculaire qui traverse l'ensemble de ses écrits.

3 Le genre le plus présent dans l'œuvre de Suárez est le policier. Ses romans s'inscrivent dans plusieurs sous-genres ou «espèces" pour reprendre le terme de Todorov: De 
cuerpo presente s'apparente au roman noir, tout comme La reina roja (1981) ou encore $E l$ síndrome de albatros (2011). El roedor de Fortimbrás (1965) et Doble Dos (1974) sont quant à eux des romans d'espionnage. Même dans des œuvres qui ne relèvent pas à part entière du genre, des éléments de celui-ci sont présents : ainsi Ciudadano Sade (2000) est un roman biographique et historique, mais dans lequel on suit, en partie du moins, l'enquête de l'inspecteur Louis Marais sur le divin marquis. L'imaginaire policier déborde le cadre de la fiction: dans son autobiographie El hombre que soñaba demasiado (2005), Suárez non seulement cite la « serie negra " parmi ses lectures de jeunesse - aux côtés de Beckett et Sartre (p. 12 et 185), mais fait appel à plusieurs reprises à la figure du détective pour se décrire lui-même (p. 42, 74, 79, 80). Ses écrits journalistiques sont aussi définis par référence au genre policier ${ }^{9}$. Notons également, bien que nous n'approfondirons pas autant cet aspect, que le fantastique est également présent dans les nouvelles de Trece veces trece (1964) et de El asesino triste (1994), ce dernier comprenant notamment une variation autour de Jekyll et Hyde, «La verdadera historia de H. y J. », et de façon plus partielle dans El roedor de Fortimbrás, Rocabruno bate a Ditirambo (1966), et Gorila en Hollywood (1980).

4 La duplicité est une caractéristique structurelle du récit policier selon Todorov, plus particulièrement du roman à énigme qu'il oppose au roman noir : «A la base du roman à énigme nous trouvons une dualité [...]. Ce roman ne contient pas une mais deux histoires : l'histoire du crime et l'histoire de l'enquête ${ }^{10}$ ». La seconde histoire a en outre pour fonction de naturaliser les procédés littéraires et les conventions qui forment la première (" pour leur donner un air "naturel" l'auteur doit expliquer qu'il écrit un livre!»), comportant un dimension fortement réflexive : «elle est souvent racontée par un ami du détective, qui reconnaît explicitement qu'il est en train d'écrire un livre : elle consiste, en somme, à expliquer comment ce récit même peut avoir lieu, comment ce livre même a pu être écrit ».

5 Bien qu'ils ne mettent pas nécessairement en place des intrigues suivant ce schéma-là et qu'ils se situent plutôt du côté du roman noir ${ }^{11}$, les récits de Suárez sont souvent marqués par la thématique du double, et par une pratique spéculaire de l'écriture ${ }^{12}$. Par-delà la division structurelle établie par Todorov, des historiens et spécialistes du roman policier soulignent la duplicité et la présence de la mise en abyme comme une caractéristique plus globale du genre. D'après Uri Eisenzweig « l'univers policier [...] est structurellement celui d'un texte : les indices sont d'ordre scriptural, l'observation est déchiffrement, le criminel se veut écrivain et le Grand Détective est avant tout un lecteur particulièrement doué13 ${ }^{13}$. Jacques Dubois consacre à son tour un chapitre à la question du "texte double ${ }^{14}$ », et Yves Reuter souligne la dimension spéculaire du roman policier ${ }^{15}$.

6 Si cette dimension spéculaire est déjà présente et caractéristique du genre policier, Suárez va l'exacerber à travers le développement de son univers personnel, de ses motifs et de ses techniques d'écriture. Étudions à présent deux de ses œuvres caractéristiques en ce sens.

7 De cuerpo presente comporte de nombreuses marques de réflexivité. D'emblée, avant même le début du texte, le livre offre une dédicace au personnage principal du roman, qui est aussi son narrateur : «A John James Nelson Braine ». Par ce premier brouillage entre les niveaux référentiels du texte, le livre se reconnait comme fiction intégrale, close sur elle-même. C'est aussi un récit impossible, l'ironie initiale tenant au fait que le narrateur se présente comme mort. La première phrase du livre est en effet «Estoy 
perfectamente muerto ». Mais cette mort n'est pourtant pas « réelle », au niveau de la diégèse du moins. Braine est un gangster ayant trahi son chef : le récit commence alors qu'il se réveille, après avoir été drogué, entouré de ses anciens compagnons, qui attendent l'arrivée du cercueil où il devra être enterré vivant. Sur ces prémisses assez classiques, le livre deviendra une folle course-poursuite après que Braine ait réussi à s'échapper du cercueil, s'écartant progressivement du modèle générique fermé pour y revenir à la fin.

8 Les références aux clichés du genre (ou généralement aux conventions fictionnelles) abondent, le texte signalant les codes qui le régissent ${ }^{16}$. Après avoir établi un précédent, qui est presque une loi du genre - " como suele ocurrir en relatos similares, es guapa » (27), dit-il à propos d'une femme qui lui ouvre sa porte - le narrateur s'adresse au lecteur lors de la seconde occurrence: "Ya imaginarán que me abre una joven. Imaginarán también que es muy guapa, por supuesto. Imaginarán, si son lectores dóciles, que la joven está sola en su casa. [...] Imaginarán todo esto, y se anticiparán a lo que yo pueda decirles. Porque, en efecto, la joven es guapa $a^{17}$ » (45). Et encore plus loin : « Es una mujer. Sí, sí. Endiabladamente bella, como suele suceder hasta la exasperación en estos relatos ${ }^{18} »(50)$.

9 Ainsi, les figures de la sérialité appartiennent à la dimension Pop de l'écriture, inspirées des pratiques industrielles tout comme l'étaient les œuvres de Warhol, et soulignent par la même occasion le mécanisme textuel. Ce ne sont pas seulement comme nous venons de le voir les rencontres de belles femmes suivies de « lo inevitable » (40, Braine couche avec elles alors qu'il n'aspire qu'au repos) qui représentent la sérialité, mais aussi l'épisode absurde de la chaîne humaine entraînée dans la chute depuis un balcon (102), ou les énumérations de morts et d'actes de violence publique dans l'avantdernier chapitre et la voracité des médias qui les couvrent (130-131). Une autre scène importante pour souligner le caractère spéculaire de l'écriture de Suárez en introduisant le motif du miroir est fondée sur la répétition, ici sous la forme simultanée du reflet. L'un des personnages rencontrés par Braine, Rod, dit être menacé par six hommes qui restent tapis dans une pièce de sa maison qu'il maintient fermée (« desde que llegué, solitario en mi coche, a este lugar, estos seis hombres, que ya me estaban esperando, no han dejado de provocarme ", 117), et qui auraient tué son meilleur ami. Il se rend dans cette pièce, qui n'est habitée que par des miroirs : ces assassins ne sont autres que son propre reflet démultiplié par six fois. Il tire sur les miroirs et meurt criblé de balles. Suite à cela, Braine confie à ses propres reflets (multipliés à l'infini par l'éclat des verres) l'histoire de Rod, la «légende " à conter et à porter "hasta los confines del mundo (Hollywood)»: « Repiten conmigo la historia, y compruebo que la han aprendido de memoria ${ }^{19} »(118)$.

10 Plus encore, l'on peut dire que les répétitions et la sérialité sont au cœur du récit : « la historia se repite » (53), observe Braine alors qu'il se retrouve de nouveau à devoir fuir en pyjama dans la rue, tenue par laquelle il finit par se faire remarque par les médias qui l'accusent d'être un violeur en série. C'est ainsi à travers la répétition que Braine devient un personnage médiatique, El Sátiro. Sa célébrité le fera rencontrer des professionnels de la culture de masses et de la reproduction sérielle : des publicitaires, des vendeurs, des journalistes, un auteur de littérature populaire... Ainsi, la répétition se produit au niveau littéral, lorsque Braine/El Sátiro devient la mascotte d'une marque de boisson fraîche dont il doit répéter le slogan ${ }^{20}$. 
11 Il y a une duplicité de la répétition, puisque celle-ci marque et impose l'identité à grande échelle et en même temps la fait vaciller dans sa remise en question de l'individualité. L'identité du personnage se définit et se dissout dans sa course, qui fait de lui un autre, une figure médiatique. Son seul but est finalement cette course qu'il ne peut plus arrêter :

Dónde voy, de dónde vengo. Me asombra que los hombres tengan tiempo en sus breves existencias de plantearse preguntas tan superfluas. Lo que importa es correr. Y lo más de prisa posible. La razón de que esté corriendo calle abajo, en vez de calle arriba, es obvia: mis piernas funcionan solas, absurdamente independientes del cuerpo de que son portadoras. ${ }^{21}$ (43)

12 Le personnage n'est qu'action ${ }^{22}$, que mouvement gratuit, celui-là même de l'écriture : «Usted es un hombre de genio, de sorprendente iniciativa. Uno de esos hombres gracias a los cuales se mueve el mundo... ( $\mathrm{Y}$ se publican los periódicos, pienso yo ${ }^{23}$ " (72). L'urgence ${ }^{24}$ et la gratuité de la course sont un reflet de la méthode d'écriture du livre, de sa logique, une mise en abyme métatextuelle pour reprendre la catégorie établie par Lucien Dällenbach, dont le principe est de réfléchir le fonctionnement du texte lui-même ${ }^{25}$. L'écriture se retrouve prise à son propre mouvement et se libère des contraintes du genre. Suárez nommera dans un livre plus tardif, mais tout aussi réflexif, cette conception de l'écriture qui s'applique aussi bien à De cuerpo presente et caractérise les rapports de notre auteur aux genres. Suárez définit en effet sa littérature comme "acción-ficción »:

No es un género nuevo. Es un género de géneros. Un género degenerado. Y, en esa medida, se parece bastante a la realidad. No como la concebimos. Unívoca. Sino como se revela a través de los medios de comunicación. Plural, informe, rabiosamente esquizoide. Ahí radica su coherencia interna. De ahí proviene su inexorabilidad lógica. Esto es lo que es y no pretende ser otra cosa. Nada de absurdo. Acontecer. Puro acontecer. Hechos que occurren, porque se me ocurren. Que suceden en el momento mismo en que suceden, tal y como suceden. ${ }^{26}$

Dans la préface à l'anthologie Las fuentes del Nilo qui recueille quelques uns de ses premiers livres, il reprend cette définition de l'écriture ${ }^{27}$, et souligne son lien au risque de la perte d'identité :

[el] acto de una escritura como acontecer en sí misma, como aventura donde imágenes y peripecias brotan de las palabras y proponen una lúdica andadura aparentemente liberada de contexto pero no extenta de la lógica del juego. Ni del peligro. Se corre, por supuesto, el riesgo de que el bosque no nos deje ver el árbol y de que, a la manera de un personaje de Conrad, o como Pulgarcito, al perder las referencias, perdamos la pista del regreso a casa. $\mathrm{O}$ la llamada identidad. Pero, ¿no es esa, precisamente, la premisa de toda auténtica aventura ?28

14 Ainsi cette pratique de l'écriture, ce "genre dégénéré », met en crise la notion d'identité. Il ne s'agit pas seulement de l'identité de l'auteur (qui est notamment problématisée dans $E l$ síndrome de albatros) : le genre est aussi un marqueur identitaire, ce qui permet d'identifier la nature d'un écrit. Cet aspect est justement très présent dans De cuerpo presente, qui insiste sur la perte d'identité de son personnage, dont toute l'existence est cette course qui perd progressivement son sens, dont la liberté s'éprouve comme pure vacance angoissante ${ }^{29}$. Mais cette crise d'identité se produit autant au niveau diégétique que méta ou transtextuel.

15 Au niveau diégétique, c'est l'identité du personnage qui subit une crise, comme nous l'avons déjà vu en analysant le rôle de la sérialité ${ }^{30}$. Mais le niveau textuel et métatextuel sont indissociables puisque l'identité du personnage est liée à celle du 
genre. Ces récriminations de son amante dans l'avant-dernier chapitre soulignent à la fois l'écart de Braine par rapport au modèle du gangster et celui du livre par rapport au roman noir :

A nadie le gusta que le vengan a complicar la existencia. Se es o no se es. Pero es necesario que los demás sepan lo que eres : zapatero, alpinista, político, gangster, ferroviario. No basta con ser Nelson Braine. [...] Con lo fácil que resulta comportarse decentemente como un gangster, jaunque sea un gangster vulgar !31 (135)

$\mathrm{Au}$ niveau transtextuel, l'inscription dans un genre populaire est contrastée et soulignée par l'intertextualité, sous forme de références et citations littéraires, dans un mélange ironique de haute et basse culture. Contrastant avec le milieu de gangsters auquel il appartient ("Hay que reconocer que tiene cultura», commente un des policiers qui cherchent à le faire avouer, 124), Braine fait référence à Descartes ("Ya lo dijo Descartes : "Pienso luego existo" ", 19), à Joyce et à Dante ${ }^{32}$, à Chateaubriand ("Las brisa marina me despeina, como si yo fuera Chateaubriand», 85), à Rimbaud (" "Par délicatesse, j'ai perdu ma vie", dijo Rimbaud», 55), et récite Baudelaire pendant un interrogatoire ${ }^{33}$. Dans cette séquence, l'on trouve également des inserts en français dont nous n'avons pu trouver l'origine, ni savoir s'il s'agit bien de citations ou d'un texte original ${ }^{34}$. Notons aussi des références à Faulkner dans le titre d'un chapitre ("Mientras agonizo ») et au sein du texte ${ }^{35}$, à L'Année dernière à Marienbad ${ }^{36}$ mais aussi des références plus populaires comme le western (un chapitre qui s'intitule « She Wore a Yellow Ribbon » comporte aussi une référence à High Noon ${ }^{37}$ ) ou Marilyn Monroe (49). Mais la référence la plus constante est celle à Shakespeare, sorte de leitmotiv de l'œuvre de Suárez ${ }^{38}$, et que Lucien Dällenbach tenait pour l'un des parangons de la mise en abyme littéraire ${ }^{39}$. La première concerne Hamlet (Elseneur est cité p. 55), puis La Tempête dans la bouche d'un publicitaire à la recherche d'un slogan pour une ligne de pyjamas : "Yo, recordando al bardo, diría que están hechos del "tejido mismo de los sueños" » (75). Le chapitre suivant, dont le titre est aussi une citation shakespearienne («Palabras, palabras, palabras»), va plus loin encore en introduisant un des personnages de Shakespeare dans la diégèse (mais à travers une parole rapportée, enregistrée, celle d'un magnétophone) : deux journalistes ont réalisé un entretien avec Hamlet, dans lequel il révèle la réalité que Shakespeare a trahi dans la pièce, souhaitant «dar una visión objetiva de mi personalidad humana » (83). Au chapitre suivant, le protagoniste reprend un monologue du prince danois pour attirer l'attention dans la rue $^{40}$. Enfin, Macbeth est cité par « el hacedor » : "Shakespeare dijo que la vida humana es un cuento narrado por un idiota » (89).

Suárez pousse la reflexivité jusqu'à introduire un personnage de romancier dans le roman. Cette rencontre de Braine avec l'écrivain, qui a lieu en deux temps, sur deux chapitres ("Y más palabras » et «El hacedor hace y deshace »), se produit au moment du désœuvrement, alors que l'action semble avoir atteint son terme, bien que nous ne soyons qu'à la moitié du livre. C'est aussi le moment du retour, qui coïncide avec ce retour du texte sur soi : après l'arrestation du (faux) Satyre, Braine n'a plus de raison de fuir, il revient vers la femme qu'il a promis d'aimer ${ }^{41}$.

Le statut réflexif de l'écrivain se transforme au fil du texte : il apparait d'abord comme un simple personnage à la même échelle que les autres, quoique ambigu et fou. Il attache et bâillonne le narrateur avec le propos de lui lire toute son œuvre. Il prétend ne pas « écrire en série ${ }^{42}$ » mais est démasqué par Braine qui l'accuse d'être plagiaire. Il avoue alors écrire les plus basses œuvres ( no desean grandes obras imprecederas, sino relatos morbosos de misterio o pornográficos. Estoy obligado a prostituirme cada día ", 
91). Ces transformations disent le caractère insaisissable de l'auteur, le texte cherchant à se saisir de son origine et à la dévoiler. Ce personnage est l'embrayeur d'une mise en abyme plus profonde et paradoxale. Dernier écrivaillon, il est aussi le premier conteur, l'imbécile originaire, le démiurge de la fable et de la vie :

Shakespeare dijo que la vida es un cuento narrado por un idiota. Pero sólo la humanidad es culpable de que la narración de ese idiota se haya convertido en una auténtica idiotez. Otros idiotas trataron sin fortuna de imitar al primer idiota, y el mundo entero se fue llenando de narraciones, plagios, vulgares plagios, pretendidamente crudos, amargos, violentos, nauseabundos "como la vida misma"... [...] Y este constante diluvio literario consiguió que, puesto que las imitaciones no lograban parecerse a la vida, fuera la vida la que acabara pareciéndose a las imitaciones. [...] ¿Usted no se ha preguntado nunca quién puede ser ese idiota a quien Shakespeare alude ? [...] Ese idiota soy yo. (89-90 ${ }^{43}$

19 Le créateur universel (" sepa que todos han pasado por aquí. TODOS $\left.{ }^{44} »\right)$ est aussi susceptible de devenir destructeur : « algun día les escupiré en la cara, pondré el punto final en las novelas de sus asquerosas vidas, porque me basta despegar la pluma del papel para que el pulso del mundo deje de latir ${ }^{45} »$ (91). Mais il s'arroge ici un pouvoir qui en fait le dépasse. Si jusqu'à présent l'on a pu prendre ses paroles pour le délire d'un fou n'engageant pas la réalité du texte, la fin du chapitre franchit un seuil de réflexivité par un détail qui relève du fantastique : Braine s'échappe de l'appartement où vit l'écrivain, mais le bruit de la machine à écrire le poursuit au loin. Alors qu'il se dirige chez la femme qu'il souhaite retrouver, et qu'il trouvera effectivement morte comme le lui avait annoncé l'écrivain ("yo la maté », lui avait-il dit), il entend à nouveau la machine dans la pièce où se trouve le cadavre. Revenant sur ses pas, il cherche a pénétrer de nouveau chez l'écrivain, mais trouve la porte fermée. Ayant réalisé son pouvoir, Braine lui demande d'arrêter d'écrire, mais il lui répond : « ¡No soy yo ! ¡Es la máquina! Escribe y escribe sola. No la puedo detener ${ }^{46} »$ (94). Pour prouver son innocence, il se suicide, tandis que la machine continue son œuvre. Braine se saisit alors de la machine et la jette dans la rue. En se fracassant au sol, elle produit une fumée en forme de champignon atomique.

Ainsi le moment le plus réflexif correspond à un moment de basculement dans le fantastique. Jusqu'ici les faits étaient restés, bien que loufoques et marqués par une frénésie proche du slapstick, dans le domaine du «réaliste». Un fait réflexif vient suspendre la réalité et marquer la nature textuelle du récit : l'entrée en scène d'une machine à écrire autonome qui consacre l'évacuation et la mort de l'auteur. La réalité dernière, la réalité même du texte, que le fantastique permet d'approcher, serait la mort. La mort est donc l'issue du texte, sa texture et sa matière même (tout comme les Pijamas El Sátiro sont « del tejido mismo de la muerte », $75^{47}$ ).

Le roman referme son cercle en revenant à la mort annoncée dès la première phrase, et plus précisément au stratagème prévu au début du livre (la bouteille de coca cola empoisonnée grâce à laquelle son patron voulait se débarrasser de Braine). Le récit se boucle sur lui-même en se terminant par la mort du narrateur qui est l'avènement et l'achèvement du texte lui-même : "Sólo tengo tiempo para comunicarles escuetamente que Nelson Braine (o sea, YO) acaba de morir. / Aquí yace para siempre, bajo este montón informe de letra impresa ${ }^{48}$ » (144). L'on peut ainsi éclairer le sens du titre, « de cuerpo presente »: au-delà du corps du protagoniste soumis à la "prueba gimnástica de la existencia humana»(110), ce corps présent serait le texte lui-même qui se manifeste dans un rapport à la mort. Les majuscules du "YO» marquent l'écart, 
l'impossible identification du narrateur avec l'auteur, le vide qui caractérise ce « moi » de l'écrivain, la mort dans laquelle il évolue, qui est son élément. A la fois affirmation et retrait, l'on peut lire la phrase en commençant par ce pronom, sans tenir compte de la ponctuation : «Yo acaba de morir », formule de l'impersonnalisation, d'un «il » qui se substitue finalement au $« \mathrm{je} »^{49}$.

La mise en abyme et la relation au genre acquièrent une dimension plus complexe dans Rocabruno bate a Ditirambo. Notons d'abord que si De cuerpo presente s'inscrivait clairement, par le milieu représenté et l'importance donnée à l'action, dans la lignée du roman noir, tout en la détournant, l'œuvre qui nous occupe à présent est plus difficile à classer. Après s'être ironiquement joué du genre, Suárez prend ses distances par rapport au roman policier. Bien qu'il contienne des éléments d'enquête, ce roman éclaté et hétérogène, "schizoïde " pour reprendre le terme employé par Suárez pour définir sa pratique de l'écriture, emprunte aussi bien au fantastique et à la sciencefiction (ou du moins au récit d'anticipation), qu'au conte, au reportage journalistique et à l'échange épistolaire.

Ici la réflexivité est marquée structurellement, au niveau de la narration elle-même. Les deux personnages-narrateurs sont des écrivains antithétiques : l'un est un journaliste naïf et consciencieux (José Ditirambo), l'autre un vieil écrivain cynique (José Rocabruno). Le fil conducteur du roman est le reportage que doit réaliser Ditirambo sur Rocabruno, mais l'instance narratrice est dédoublée, le livre alternant les chapitres écrits par l'un et par l'autre. Dans ce chassé-croisé, les deux auteurs se mettent mutuellement en scène, brouillant les cadres du fictif et du véridique: dans l'un des reportages de Dititrambo, "La Caperucita negra ", Rocabruno apparaît sous les traits du loup garou, reportage que l'écrivain, dans le chapitre suivant, dénonce comme un plagiat de son récit "La Caperucita rubia ", auquel le reportage fait justement suite dans l'ordonnancement des chapitres. Le récit principal et central du livre, celui d'un meurtre à venir que Ditirambo doit déjouer dans une Barcelone menacée par l'éveil du volcan Tibidabo, possiblement provoqué par une série d'essais nucléaires souterrains, est présenté en deux versions, la première sous forme d'une "fable " écrite par Rocabruno ( La Caperucita rubia »), qui se présente à son tour comme le «relato de una película ${ }^{50}$ ", et la seconde narrée par Ditirambo ("Ditirambo investiga ») qui actualise ce récit après sa rencontre avec Rocabruno, et après avoir lu la lettre que celui-ci lui a remis à l'intérieur d'une bouteille, signée par l'un des personnages de la fable (son nom apparaît sous forme d'acrostiche, codage ayant déjà été employé dans un des reportages-enquêtes de Ditirambo).

Le livre joue sur une autre forme de l'intertextualité que De cuerpo presente, il opère davantage par la réécriture, à travers les variations autour de cet emblème de la fabulation qu'est l'histoire du petit chaperon rouge, mais aussi par le collage, qui est ici incursion de la réalité dans la fiction et vice versa : l'insertion de coupures de presse ${ }^{51}$ influe sur le cours du récit, les personnages les manipulent ou les interprètent pour faire avancer l'action.

Le mélange de genres et des niveaux de réalité participe de ce trouble de l'identité propre à l'écriture. À la fin, les différents niveaux du récit se confondent ${ }^{52}$, et la mort semble être de nouveau la clé de l'énigme et la réalité dernière : Ditirambo, perdu dans ses incertitudes, dit « Por un momento, me encontré tan indefenso que sentí deseos, no lo oculto, de echarme a llorar en brazos del cadáver ${ }^{53}$ » (470), et dans le chapitre final, il voyage dans un train où tous les passagers sont morts. Rocabruno les tranforme en 
conteurs par un exercice de ventriloquie, la dernière narration révélant sa supercherie étant précisément une histoire de fantômes, un récit fantastique proche de ceux de Trece veces treces où la causalité s'inverse ${ }^{54}$.

Tout comme dans De cuerpo presente, le roman accomplit une boucle sur lui-même, commençant par un récit onirique de Rocabruno dans une lettre adressée à la femme à laquelle il devait, dans son rêve, remettre une enveloppe («acabo de comprender [...] que el contenido del sobre es precisamente esta carta que estoy escribiendo en estos momentos ${ }^{55} "$, 371), et se terminant par l'inclusion de Rocabruno et Ditirambo dans le décor et l'action de ce rêve. Cette boucle marque l'identité des deux personnages, le roman se terminant par l'entrée des deux écrivains dans une pièce où se trouve un personnage encapuché : « Usted soy yo - dijo el escritor José Rocabruno quitándose el capuchón ante el espejo ${ }^{56} »(487)$.

Ici la spécularité fonctionne donc à plein régime, mêlant les identités des genres autant que celles des personnages, rejouant au niveau de sa construction le thème fantastique du double. Elle est soulignée également par diverses références au miroir : outre le fait que le dernier mot du livre soit « espejo ", l'on retrouve, dans une lettre de Rocabruno adressée à un directeur de théatre, un projet de pièce qui consisterait à placer un miroir devant le public, celui-ci se rendant progressivement compte du dispositif dans lequel il est pris (388-389). Des paroles de chanson sont inversées par la curieuse acoustique d'un entrepôt ("Los techos de las naves [...] actuaban como un espejo", 400), et Rocabruno transformé en loup garou dit de façon énigmatique : «Si hubiera algún espejo, todo quedaría explicado $»^{57}$ (425). Enfin, lorsque Ditirambo évoque l'intérêt des lecteurs pour le reportage qu'il pense lui consacrer, l'écrivain répond : "¿A qué lectores?", et regarde avec inquiétude autour de lui (442).

L'on voit donc ici que les mises en abyme sont génératrices du récit - alors que dans $D e$ cuerpo presente elles étaient plutôt produites par le mouvement frénétique de l'écriture cherchant à se ressaisir elle-même dans des signes réflexifs plus épars, et dans la sérialité - que le roman est bâti sur la spécularité, qu'il lui doit sa structure, cas analogue à celui des Lieux-dits de Jean Ricardou où selon Dällenbach la mise en abyme devient productive et consacre l'autonomie du texte (Dällenbach : 207). La victoire du roman sur le guide touristique chez Ricardou (182) fait écho à celle de l'écrivain sur le journaliste soulignée par le titre de Suárez.

29 Ainsi, l'œuvre de Gonzalo Suárez entretient avec le policier une relation analogue à celle des nouveaux romanciers, faisant d'un genre codifié une base assez rigide, reconnaissable et pour ainsi dire neutre, pour marquer l'exploration du texte par luimême et l'expérimentation romanesque ${ }^{58}$. Suárez met en place une généricité réflexive, crée un "genre de genres». L'appel au marqueur d'identité qu'est le genre littéraire devient un instrument réflexif, fait apparaître une écriture spéculaire où se déploie le thème du double, multipliant les jeux de miroir entre la réalité et la fiction, mêlant la convention et l'invention. L'énigme qui caractérise le récit policier se tourne vers sa propre production, et vers le rapport qu'elle entretient avec une dimension originelle liée aussi bien à la mort qu'à l'érotisme (dimension développée à partir des années 80 dans des romans d'inspiration policière tels que La reina roja ou El síndrome de albatros), qui fait dialoguer les textes avec le fantastique. L'écriture-action défait ainsi les genres tout en les employant, les mélange dans un «genre dégénéré ». 


\section{BIBLIOGRAPHIE}

BLANCHOT, Maurice, L'éspace littéraire, Paris : Gallimard, 1993.

CERCAS, Javier, La obra literaria de Gonzalo Suárez, Barcelona : Quaderns Crema, 1993.

DÄLLENBACH, Lucien, Le récit spéculaire : essai sur la mise en abyme, Paris : Seuil, 1977.

DUBOIS, Jacques, Le roman policier ou la modernité, Paris : Nathan, 1992.

EISENZWEIG, Uri, Le récit impossible, Paris : C. Bourgois, 1986.

FAULKNER, William, Snopes: The Hamlet, The Town, The Mansion, New York : Modern Library, 2011.

JANVIER, Ludovic, Une parole exigeante : Le nouveau roman, Paris : Editions de Minuit, 1970.

REUTER, Yves, Le roman policier, Malakoff : Armand Colin, 2017.

SALAGRIDAS, « Doble Dos: ¿política-ficción ? », El Mundo, 08/02/75.

SUÁREZ, Gonzalo, Entretien avec René Micheaux, « Un monstruo de nuestro tiempo », Mistery

Magazine, janvier 1965.

SUÁREZ, Gonzalo, La reina roja, Madrid : Cátedra,1981.

SUÁREZ, Gonzalo, El hombre que soñaba demasiado, Barcelona : Plaza \& Janés, 2005.

SUÁREZ, Gonzalo, Las fuentes del Nilo, Madrid : Alfaguara, 2011.

TODOROV, Tzvetan, Poétique de la prose, Paris : Seuil, 1971.

\section{NOTES}

1. Bien que Suárez refuse l'appartenance à toute école : «Au début des années 60 , je cherchais seulement la liberté de faire du cinéma. C'était l'époque, pédante et adolescente, de la « Nouvelle Vague » et de «L'école de Barcelone ». Je ne me suis jamais rattaché aux modes ni aux tendances. Les dynamiques de groupe me répugnent. Je ne supporte pas l'esprit corporatif et grégaire de ceux qui veulent uniformiser des opinions pour faire la ronde ", El Mundo, 7 décembre 1996.

2. Gonzalo Suárez, Las fuentes del Nilo, Alfaguara, 2011, quatrième de couverture.

3. Robert Saladrigas, « Doble Dos: ¿política-ficción?», El Mundo, 08/02/75, p. 46.

4. Javier Cercas, La obra literaria de Gonzalo Suárez, Quaderns Crema, 1993, p. 14. Sur son manque de succès commercial, Suárez raconte l'anecdote suivante: «De mon premier roman publié, De cuerpo presente, l'on avait distribué 80 exemplaires à Madrid dont 81 ont été retournés. Un grand succès, si l'on considère que le précédent, un pavé de 500 pages, avait été entièrement rejetté par la censure ", Gonzalo Suárez, El hombre que soñaba demasiado, Plaza \& Janés, 2005, p. 202.

5. "J'estimais que les chefs d'œuvres et leurs succédanés meublaient nos maisons, mais lesdits genres mineurs nous ouvraient les fenêtres », Op. cit. p. 13.

6. Dans la préface à La reina roja (1981), Suárez se réfère à la réalité « como se revela a través de los medios de comunicación » comme son modèle d'écriture.

7. «cette attitude du pop art est l'attitude dont part fondamentalement l'œuvre de Gonzalo Suárez; notre auteur utilise les référents et stylèmes de la culture de masses, les déforme et les soumet à une critique ironique mais implacable afin de se confronter à l'environnement d'une civilisation dominée par cette nouvelle culture propagée par les mass media », Op. cit., p. 252. 
8. Entretien avec René Micheaux, "Un monstruo de nuestro tiempo », Mistery Magazine, janvier 1965, p. 7.

9. « Entre sus influencias como reportero, Suárez cita a dos grandes de la novela negra, Dashiell Hammett y Raymond Chandler. "El periodismo me permitía aceptar la realidad con el subterfugio de jugar a detective", asegura. ", https://elpais.com/diario/2006/04/08/cultura/ 1144447207_850215.html. Il mentionne également son goût pour Hammett, Cheyney et Chandler dans un entretien récent : http://www.elcultural.com/noticias/letras/Gonzalo-Suarez-Paris-enlos-50-era-un-deseo-pero-tambien-tenia-una-oscura-trastienda/7434

10. Tzetvan Todorov, Poétique de la prose, Seuil, 1971, p. 57.

11. «Le roman noir est un roman policier qui fusionne les deux histoires ou, en d'autres mots, supprime la première et donne vie à la seconde. Ce n'est plus un crime antérieur au moment du récit qu'on nous relate, le récit coïncide maintenant avec l'action » (Ibid). Selon Todorov le roman se caractérise notamment par son contenu thématique. Au niveau des personnages, Yves Reuter évoque ainsi les «types mythologiques de notre culture : loser, privé, femme fatale ou vamp, tueur, policier corrompu, politicien véreux... » (Yves Reuter, Le roman policier, Armand Colin, 2017, p.62) tout en soulignant la diversité du genre et la liberté qui peut être prise envers ces archétypes. Un autre élément caractéristique est la complexité ou confusion de l'intrigue, que nous retrouverons bien dans La reina roja et El síndrome de albatros : "La figure emblématique de ce genre serait plutôt l'embrouille, l'imbroglio que l'on va tenter de démêler, souvent à l'aveuglette ", (Ibid., 57).

12. La spécularité est à ce point indissociable du récit que dans l'une des nouvelles de Gorila en Hollywood, "Espejo", c'est un miroir qui prend la parole, racontant à une assemblée de « confrères » tout ce qu'il a vu et reflété au cours de sa vie.

13. Uri Eisenzweig, Le récit impossible, C. Bourgois, 1986, p. 154

14. Jacques Dubois, Le roman policier ou la modernité, Nathan, 1992, p. 77-81.

15. Op. cit., p. 109-110.

16. «- Henry tiene un complejo, ¿sabe ? No me extraña. En la vida todos tienen complejos, varios. En las películas o en las novelas, los personajes sólo tienen un complejo, un único complejo que justifica todo lo que hacen y lo que dejan de hacer. Henry debe de ser un personaje de novela » (Las fuentes del Nilo: 51) («Henry souffre d'un complexe, vous savez? / - Ca ne m'étonne pas. Dans la vie toute le monde a des complexes, plusieurs. Dans les films ou les romans, les personnages n'ont qu'un seul complexe, un complexe unique qui justifie tout ce qu'ils font et ce qu'ils cessent de faire. Henry doit être un personnage de roman »), «Lo ha oído en cien películas, lo ha leído en cien novelas. Lo dice de una manera muy convincente » (62) ("Elle l'a entendu dans cent films, elle l'a lu dans cent romans. Elle le dit d'une façon très convaincante »), "También hemos previsto el caso, nada improbable, de que usted acabe acribillado a balazos en cualquier plaza pública. ¡Como en las películas!»(72) («Nous avons aussi prévu le cas, nullement improbable, où vous vous retrouveriez criblé de balles dans n'importe quelle place publique. Comme dans les films!»)

17. «Vous imaginerez que c'est une jeune femme qui m'ouvre la porte. Vous imaginerez aussi qu'elle est belle, évidemment. Vous imaginerez, si vous êtes des lecteurs dociles, que la jeune femme se trouve seule chez elle. [...] Vous imaginerez tout cela, et vous anticiperez ce que je pourrais vous dire. Parce que, en effet, la jeune femme est belle »

18. «C'est une femme. Oui, oui. Diaboliquement belle, comme cela arrive à une fréquence exaspérante dans ce genre de récits »

19. «Ils répètent l'histoire avec moi, et je constate qu'ils l'ont apprise par cœur »

20. « la casa "ROSINCOLA" pone a su disposición la cantidad de diez mil quinientos dólares si usted se compromete, mediante el presente contrato, a responder a las preguntas de la policía y de los periodistas con una sola e inalterable frase : "BEBA ROSINCOLA Y VIVA MEJOR" » (72) (« la marque "ROSINCOLA" met à votre disposition une quantité de dix mille cinq cent dollars si vous 
vous engagez, par le présent contrat, à répondre aux questions de la police et des journalistes par une seule et inaltérable phrase : "BUVEZ ROSINCOLA ET VIVEZ MIEUX" »).

21. "Où je vais, d'où je viens. Je suis ébahi que les hommes aient le temps dans leurs brèves existences de se poser des questions si superflues. Ce qui compte c'est de courir. Et le plus vite possible. La raison pour laquelle je descends la rue en courant, plutôt que de la remonter, est évidente: mes jambes fonctionnent seules, absurdement indépendantes du corps qu'elles portent»

22. Le roman noir est résolument tourné vers l'action, contrairement au roman à énigme : «Dans le roman noir, la violence et les actions ont une place essentielle ", Yves Reuter, Op. cit., p. 63. Cela est manifeste au niveau même de la structure narrative : « le récit coïncide maintenant avec l'action », comme le soulignait Todorov (Op. cit.).

23. "Vous êtes un homme de génie, d'une initiative étonnante. L'un de ces hommes grâce auxquels le monde bouge... (Et les journaux sont publiés, je pense)»

24. «EMERGENCIA. Maravillosa y dúctil palabra. [...] Si algún día me dejan el tiempo necesario de enamorarme (enamorarse es una cuestión de ocio), me enamoraré de una palabra y no de una mujer. No debemos olvidar que sin palabras no existiría el amor. Ni los periódicos de la mañana » (70) («URGENCE. Merveilleuse et ductile parole. [...] Si un jour on me laisse le temps nécessaire pour tomber amoureux (l'amour est question de loisir), je tomberai amoureux d'un mot et non d'une femme. Il ne faut pas oublier que sans les mots l'amour n'existerait pas. Ni les journaux du matin »).

25. Lucien Dällenbach, Le récit spéculaire, Seuil, 1977, p. 123-131.

26. Gonzalo Suárez, La reina roja, Cátedra, 1981, p. 9. «Ce n'est pas un nouveau genre. C'est un genre de genres. Un genre dégénéré. $\mathrm{Et}$, à ce titre, il ressemble assez à la réalité. Pas telle qu'on la conçoit. Univoque. Mais telle qu'elle se révèle à travers les moyens de communication. Plurielle, informe, rageusement schizoïde. C'est cela qui fonde sa cohérence interne. C'est de là que vient son inexorable logique. C'est ce que c'est et ça ne prétend pas être autre chose. Rien d'absurde. De l'événement. Du pur événement. Des faits qui arrivent parce qu'ils me viennent à l'esprit. Qui se produisent au moment même où ils se produisent, tels qu'ils se produisent »

27. «Opté por escribir libros que asumieran su condición real. Es decir, no serían verdades de mentira sino mentiras de verdad, cosas que ocurrían porque se me ocurrían y que petulantemente, traté de homologar como género y di en llamar acción ficción » (14). («J'ai choisi d'écrire des livres qui assumeraient leur condition réelle. C'est-à-dire qu'ils ne seraient pas des vérités factices, mais de vrais mensonges, des choses qui arrivaient parce qu'elles me passent par l'esprit et que, de façon pétulante, j'ai tenté d'homologuer comme genre et que j'ai nommé action-fiction »)

28. Id. « ( $\left(l^{\prime}\right)$ acte d'une écriture comme événement en elle-même, comme aventure où les images et péripéties naissent des mots et proposent un parcours apparemment libre de tout contexte mais non dépourvu de la logique du jeu. Ni du danger. L'on court le risque, bien entendu, que la forêt nous cache l'arbre et que, à la façon d'un personnage de Conrad, ou comme le Petit Poucet, en perdant les références, l'on perde la piste du retour chez soi. Ou ce que l'on appelle l'identité. Mais, n'est-ce pas là précisément la prémisse de toute aventure authentique ?»

29. "Yo corro, al fin, en algun sentido. $Y$ voy y vengo. $Y$ la desesperación va dilatándose dentro de mi. Es como un globo. Un globo que debo mantener desesperadamente hinchado si no quiero quedar automáticamente muerto » (87) («je cours, à la fin, dans n'importe quelle direction. Je vais et viens. Et le désespoir se dilate en moi. C'est comme un ballon. Un ballon qui doit rester désespérément gonflé si $\mathrm{j}$ ne veux pas me retrouver automatiquement mort "), "Yo era completamente libre. ¿Qué podía hacer con mi libertad?» (138) («J'étais complètement libre. Que pouvais-je faire de ma liberté ?»).

30. Même la figure du Satyre est soumise à la sérialité. Lorsque Braine appelle la police pour se dénoncer, pensant atteindre le repos tant désiré dans l'incarcération, celle-ci arrive jusqu'à 
l'immeuble où il se trouve et lui demande de se livrer : Braine voit alors sortir « cabizbajos, en fila india, uno, dos, tres, cuatro y hasta siete hombres uniformados con pijamas a rayas » répondant au singalement donné ( Sabemos que está usted en la casa. Sabemos que viste un pijama a rayas. Lo sabemos todo"(61). Alors que Braine essaie désespérément de se livrer à la police, c'est finalement un « usurpateur» qui sera capturé comme l'annoncent les journaux (84). L'un des journalistes qui voulait l'interviewer lui lance à ce moment: "Ahora ya no es usted nadie, NADIE » (85) (« Vous n'êtes plus personne à présent, PERSONNE »).

31. «Personne n'aime qu'on lui complique l'existence. On est ou on n'est pas. Mais il faut que les autres sachent ce que tu es : cordonnier, alpiniste, politicien, gangster, cheminot. Ce n'est pas suffisant d'être Nelson Braine. [...] Alors qu'il est si facile de se comporter décemment comme un gangster, même si c'est un gangster vulgaire!»

32. « la chaqueta me caería bien si consiguiese mantener debajo, pegado a la espalda, un ejemplar del Ulises, de James Joyce, con La Divina Comedia a modo de epílogo » (26), « el cañon de una pistola se obstina en introducirse entre mis costillas. Precisamente en el lugar donde debiera haber colocado el Ulises de James Joyce, con la absoluta garantía de que ni las balas pasan la página tercera » (29).

33. «Entonces les recito un poema de Baudelaire, y no les gusta. [...] Pero, claro, a ellos no les gusta lo que a mí me gusta », p. 123.

34. «Ce ne fût [sic] pas la négation d'un esprit, mais d'un sprit [sic], avec des conditions préalables d'expansion de matière au sein du vide, de vibration, d'osmose, d'attraction, de contact, de réflexe, de gérmination [sic], d'éclosion de vitalité... [...] La destruction, la bléssure [sic], la crasse, la lézarde, la tache... », p. 122-123.

35. La référence s'accompagne de ce qui semble être une citation, dans le cadre d'un concours auquel participe le protagoniste, présentant des affinités avec le collage qui serait pratiqué de façon plus systématique dans Rocabruno bate a Ditirambo: «ALTAVOZ: ¿Cómo se llamaba el protagonista de El villorrio, la famosa novela de William Faulkner - No se torturaba con una impotente amargura recordando, ni rechinaba los dientes, porque los dientes no le rechinaban. Tal vez buscaba tan sólo la oferta del espacio ilimitado y del irremediable olvido... »(67). Cependant d'après nos recherches ce passage et les suivants que nous ommettons ici n'appartiennent pas littéralement au roman de Faulkner, mais en sont plutôt des réécritures ou des pastiches, qui reprennent certains motifs ou fragments de l'original : ainsi l'on retrouve par exemple le passage "Perhaps he was seeking only the proffer of this illimitable space and irremediable forgetting" (William Faulkner, Snopes: The Hamlet, The Town, The Mansion, Modern Library, 2011, p. 222) mais pas de références aux dents serrées - du moins pas dans le voisinage immédiat de cette phrase.

36. «Tengo la sensación de haber pasado ya antes bajo estos techos. De haber recorrido ya antes estos pasillos. ¿Cuándo? ¿Dónde? ¿El año pasado? ¿En Nueva York? ¿En Toulouse? ¿En Barcelona? ¿En Marienbad?» (71).

37. « Tengo miedo, sí. Pero, ¿no tenía miedo Gary Cooper en Solo ante el peligro ?» (117).

38. On retrouve cela aussi dans El roedor de Fortimbrás, dans l'épigraphe de Doble Dos et dans Gorila en Hollywood où il propose une réécriture, «El auténtico caso del joven Hamlet », qu'il portera ensuite à l'écran dans le film Epilogo (1984).

39. Op. cit., p. 16. Les références sont d'ailleurs empruntées majoritairement à Hamlet, dont Dällenbach affirme : «De toutes les duplications intérieures de l'histoire de la littérature, nulle n'est plus fameuse que la "pièce dans la pièce" de Hamlet ", Ibid., p.22.

40. "¿Quién sufriera del tiempo la irrisión y vil escarnio, del opresor el yugo, los ultrajes del orgulloso, el ansia, los tormentos de un mal pagado amor, de la justicia la lentitud, del mando la insolencia, el menosprecio con que trata

la indignidad al mérito paciente? [...] ¿Quién soportara gravosas cargas, quién gimiera 
triste, sudando bajo el peso de esta odiosa, cansada vida... ? [...] Así en cobardes nos convierte a todos tremenda la conciencia... » (86).

41. «El abajo firmante, cuyo nombre desconozco, se compremete a enamorarse de mí, si logra escapar con vida de la aventura en que, por causas ajenas a su voluntad, se ve envuelto » (62) («Le signataire, dont j'ignore le nom, s'engage à tomber amoureux de moi, s'il parvient à réchapper vivant de l'aventure où, pour des raisons étrangères à sa volonté, il se trouve impliqué »).

42. «Soy hacedor de libros. Comedias. Divinas o humanas. Lo que quiera. Trabajadas a mano. Jamás escribo en serie », (88) («Je suis faiseur de livres. Des comédies. Divines ou humaines. Selon votre goût. Faites à la main. Je n'écris jamais en série »).

43. "Shakespeare a dit que la vie est un conte raconté par un idiot. Mais seule l'humanité est responsable du fait que le récit de cet idiot soit devenu une idiotie réelle. D'autres idiots ont tenté sans succès d'imiter le premier idiot, et le monde entier s'est progressivement rempli de récits, plagiats, vulgaires plagiats, prétendument crus, violents, nauséabonds « comme la vie même »... [...] Et ce constant déluge littéraire a fait que, puisque les imitations n'arrivaient pas à ressembler à la vie, ce fut la vie qui a fini par ressembler aux imitations. [...] Vous ne vous êtes jamais demandé qui pouvait être cet idiot auquel Shakespeare se réfère ? [...] Cet idiot c'est moi. »

44. «Sachez que tous sont passés par là. TOUS »

45. «Un jour je leur cracherai au visage, je mettrai un point final aux romans de leurs vies répugnantes, parce qu'il me suffira de détacher la plume du papier pour que le pouls du monde cesse de battre »

46. «Ce n'est pas moi ! C'est la machine ! Elle écrit et continue d'écrire toute seule. Je ne peux pas l'arrêter »

47. Braine dit aussi à l'écrivain : « ¿Sabe lo que es lo más profundo ? [...] ¡La tumba ! ¡La muerte! » (89) (« Vous savez ce qui est le plus profond ? [...] La tombe ! La mort ! »).

48. "J'ai seulement le temps de vous communiquer brièvement que Nelson Braine (c'est-à-dire, MOI) vient de mourir. / Il gît pour toujours sous cet amas informe de lettres imprimées »

49. Maurice Blanchot a exprimé cette impersonnalisation de l'écriture : «Le "Il" qui se substitue au "Je", telle est la solitude qui arrive à l'écrivain de par l'œuvre. [...] "Il", c'est moi-même devenu personne, autrui devenu l'autre, c'est que, là où je suis, je ne puisse plus m'adresser à moi et que celui qui s'adresse à moi, ne dise pas "Je", ne soit pas lui-même " (L'espace littéraire, Gallimard, 1993, p. 23), ainsi que les liens de l'écriture et de l'écrivain à la mort : «il est lié à l'œuvre de la même manière que l'est à la mort l'homme qui la prend pour fin ", Ibid., p. 132.

50. Cette histoire est effectivement la reprise du scénario écrit par Suárez pour le film de Vicente Aranda, Fata Morgana (1965), dont la réalisation ne le laissa pas satisfait.

51. Vraisemblablement authentiques, puisque Suárez mentionne dans les remerciements qui précédent le texte « les journaliste anonymes qui quotidiennement m'ont fourni les nouvelles utilisées au long du récit » (368). Le titre du livre pourrait lui-même être tiré de l'actualité sportive, Rochebrune et Ditirambo étant originellement des chevaux de course comme l'indique l'auteur dans cette même préface. L'on trouve déjà quelque chose d'analogue dans De cuerpo presente, quoique moins systématique : ces coupures relèvent plutôt de l'imitation, relatant les événements du livre $(70,84,131)$, mais celle annonçant la mort de Marylin Monroe pourrait être authentique (65).

52. «En realidad no sabía si me hallaba a punto de finalizar el más sensacional y verídico reportaje de José Ditirambo, o si más bien se trataba del último relato del escritor José Rocabruno, o ni siquiera eso, y quizá todo fuera tan sólo producto de los perniciosos efectos del alcohol sobre el cerebro de un niño tonto » (469) («En réalité je ne savais pas si j'étais sur le point de terminer le plus sensationnel et véridique reportage de José Ditirambo, ou s'il s'agissait plutôt du dernier récit de l'écrivain José Rocabruno, ou pas même cela, et peut-être tout n'était-il que le produit des pernicieux effets de l'alcool sur le cerveau d'un enfant idiot »). 
53. « Pour un moment, je me suis senti si vulnérable que j'ai eu, je ne le cache pas, envie de me mettre à pleurer dans les bras du cadavre »

54. «En realidad, las causas son residuos de las consecuencias " (« en réalité, les causes sont des résidus des conséquences »), " $\mathrm{Al}$ volver de la zeta », Trece veces trece, in Las fuentes del Nilo, p. 231. Rocabruno parle à un autre endroit $\mathrm{d}$ '« una obra en la que la causa y el efecto se producían de manera simultánea y paralela » (445) (« une œuvre où la cause et l'effet se produisaient de façon simultanée et parallèle »).

55. « je viens de comprendre [...] que le contenu de l'enveloppe est précisément cette lettre que je suis actuellement en train d'écrire » (371)

56. «Vous êtes moi - dit l'écrivain José Rocabruno en enlevant sa capuche face au miroir »

57. «S'il y avait un miroir quelque part, tout serait expliqué »

58. Ludovic Janvier, Une parole exigeante, Editions de Minuit, 1970, p. 49. Javier Cercas voit aussi en Suárez un précurseur dans le champ de la littérature espagnole quant à l'usage des «schémas et atmosphères du genre policier ", qui s'est développé dans le seconde moitié des années 70 - et même avant dans l'œuvre de Cargenio Trías, Santa Ava de Adis Abeba, où il reconnaît l'influence de De cuerpo presente - dans des « projets de romans littérairement ambitieux et artistiquement exigeants, mais soucieux du lien post-moderne que constitue le pacte avec grande majorité des lecteurs » (Darío Villanueva). Cercas cite à ce titre Manuel Vázquez Montalbán, Juan Benet, Juan Marsé, Fernando Savater, Eduardo Mendoza, Juan Madrid, Juan José Millás, Soledad Puértolas, Antonio Muñoz Molina, tout en reconnaissant la diversité de leurs esthétiques (Op. cit. p. 169-170, voir aussi p. 320-325).

\section{RÉSUMÉS}

L'œuvre littéraire de Gonzalo Suárez n'appartient pas réellement au champ de la littérature populaire, mais emprunte à celle-ci et notamment au policier et au fantastique, pour élaborer une écriture marquée par la réfléxivité et le dédoublement. Plus encore, sa démarche a été définie comme celle d'un "écrivain pop ». Nous étudierons la dimension spéculaire de deux de ses premiers romans, De cuerpo presente (1963) et Rocabruno bate a Ditirambo (1966), leur façon de s'approprier et de détourner les codes du genre, et de remettre en question la notion même de genre.

\section{INDEX}

Mots-clés : littérature espagnole, mise en abyme, Gonzalo Suárez, littérature populaire, XXème siècle, littérature policière, genres littéraires

\section{AUTEUR}

\section{BORIS MONNEAU}

Programmateur et critique de cinéma

boris.monneau@hotmail.fr 